\title{
Analytical STEM Study of P-Doped Silicon Nanocrystals Exhibiting Mid-Infrared Localized Surface Plasmon Resonance
}

\author{
J.S. Jeong ${ }^{1}$, D.J. Rowe ${ }^{2}$, U.R. Kortshagen ${ }^{2}$ and K.A. Mkhoyan ${ }^{1}$ \\ ${ }^{1}$ Chemical Engineering and Materials Science, University of Minnesota, Minneapolis, MN 55455, USA \\ ${ }^{2}$ Mechanical Engineering, University of Minnesota, Minneapolis, MN 55455, USA
}

Localized surface plasmon resonance (LSPR) is the collective oscillation of free charge carriers in nanometer-sized materials by incident light [1]. Nanocrystals exhibiting LSPR, by an external electromagnetic field, present remarkable light scattering and absorption phenomena which provide access to their applications in bio-sensing, spectroscopy enhancement, sub-wavelength microscopy, and photovolatics. Since metals have large free charge carrier concentration, resulting in resonances within a visible range, significant attention has been given to noble metals. Additionally, recent work has provided the potential of heavily doped semiconductor nanocrystals for LSPRs in the infrared range, a region of the spectrum less accessible to metal nanocrystals [2,3]. Nevertheless, no LSPRs have been reported for doped silicon nanocrystals (SiNCs) despite silicon's importance in electronic and photonic applications. The difficulty of realizing LSPRs in SiNCs is primarily associated with effectively doping semiconductor nanocrystals [4]. Active doping is often limited by phase segregation of dopants during synthesis, inactive dopants trapped in the structural defects, and dopant incorporations in improper sites, leading to insufficient free carrier concentrations for LSPRs in SiNCs, which demand extensive atomicresolution microscopy and spectroscopy studies about dopant distributions and electronic structures of dopants.

Here we demonstrate tunable LSPRs in the range of 0.07-0.3 eV in phosphorus (P)-doped SiNCs, which are produced using a nonthermal plasma synthesis approach (Figure 1) [5]. FTIR measurements are performed in a nitrogen purged glovebox at room temperature. For (scanning) transmission electron microscopy ((S)TEM) studies, SiNCs in a sub-monolayer are directly prepared onto copper TEM grids covered with a holey carbon supporting film. The samples are then transferred into a microscope under minimal ambient exposure. Characterization of SiNCs, including high-resolution TEM (HRTEM) and annular dark-field (ADF) STEM imaging, and energy dispersive x-ray spectroscopy (EDX) is conducted using an FEI Tecnai G2 F-30 (S)TEM operated at accelerating voltages of 300 and $200 \mathrm{kV}$.

TEM analyses rule out formation of unwanted byproducts like silicon phosphides under a high fractional $\mathrm{PH}_{3}$ flow rate, defined as $X_{P H 3}=\left[\mathrm{PH}_{3}\right] /\left(\left[\mathrm{PH}_{3}\right]+\left[\mathrm{SiH}_{4}\right]\right) \times 100 \%$, up to 16 at $\% \mathrm{P}$ incorporation into $\mathrm{SiNCs}$. HRTEM reveals that as-produced SiNCs with minimal air exposure have a crystalline core, even at the highest doping concentrations, with approximately $1 \mathrm{~nm}$-thick amorphous shell, regardless of $\mathrm{P}$ doping level. The size of SiNCs strongly decreases with increasing P doping as confirmed by size determination via X-ray diffraction and TEM (Figure 2); moreover, the smaller SiNCs are the more twins they have. These grain boundaries from twining result in an increasing number of plasmon scattering interfaces, explaining the observed LSPR broadening at large $X_{P H 3}$. STEM EDX measurements suggest that $\mathrm{P}$ is either incorporated into the SiNCs and/or condensed on the SiNC surfaces (Figure 3); however, the presence of surface Si-P $x$-Hy vibrations (shown in Figure 1) indicates significant $\mathrm{P}$ segregation at the surface. HF vapor etching experiments to remove surface oxide shells and so do surface P suggest that $60-70 \%$ of $\mathrm{P}$ was condensed on the SiNCs surface (Figure 4); however, SiNCs still contain enough core $\mathrm{P}$ dopants to exhibit LSPRs. The amorphous shell observed in TEM is not fully explained by amorphous 
silicon oxides, but instead P-induced amorphous matrix or structural disorder that is a byproduct of the plasma synthesis process or intrinsic property of nanocrystals with high surface-to-volume ratio.

\section{References:}

[1] U. Kreibig and M. Vollmer in "Optical Properties of Metal Clusters", (Springer, Berlin).

[2] J.M. Luther et al., Nat. Mater. 10 (2011), 361.

[3] K. Manthiram and A.P. Alivisatos, J. Am. Chem. Soc. 134 (2012), 3995.

[4] D.J. Norris, A.L. Efros and S.C. Erwin, Science 319 (2008) 1776.

[5] L. Mangolini, E. Thimsen and U.R. Kortshagen, Nano Lett. 5 (2005), 655.

[6] This work was supported primarily by the MRSEC program of the National Science Foundation under Award Number DMR-0819885.

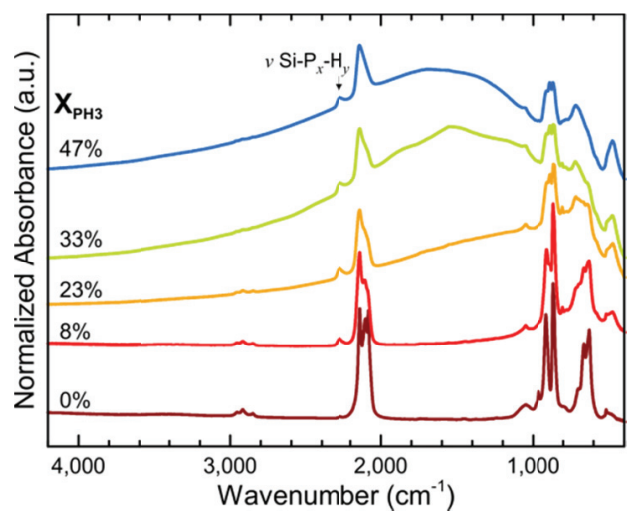

Figure 1. Normalized FTIR spectra of Pdoped SiNCs for a range of $X_{P H 3}$, showing a broad absorption consistent with LSPR shifts.

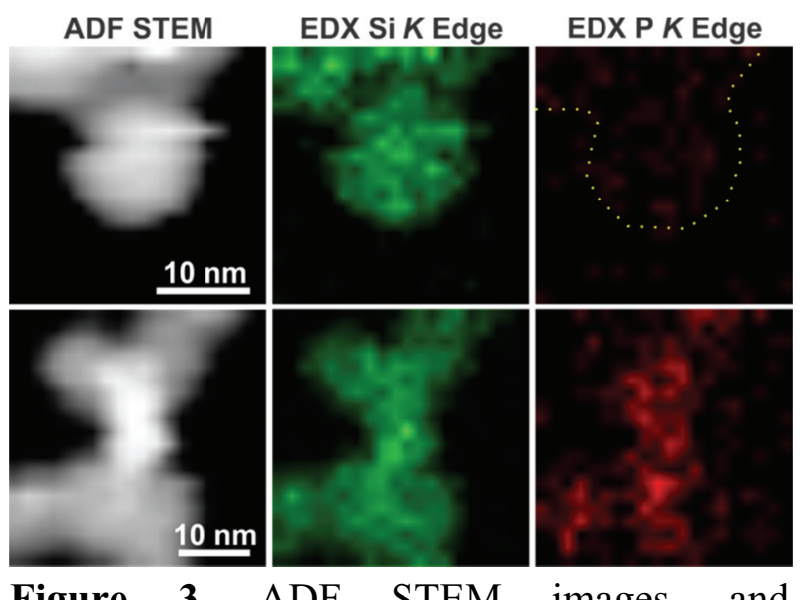

Figure 3. ADF STEM images, and corresponding EDX maps of Si $K$ and $\mathrm{P} K$ edges recorded in parallel from undoped (top panels) and heavily P-doped (bottom panels) SiNCs.

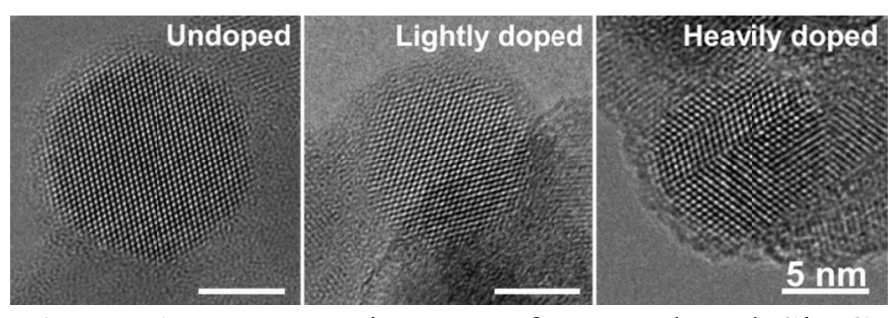

Figure 2. HRTEM images of as-produced SiNCs with different $\mathrm{P}$ doping level: undoped, lightly, and heavily doped $\left(X_{P H 3}=0 \%, 8.2 \%\right.$, and $47 \%$, respectively). SiNCs have a crystalline core with $\sim 1$ $\mathrm{nm}$-thick amorphous shell, regardless of the $\mathrm{P}$ doping level.

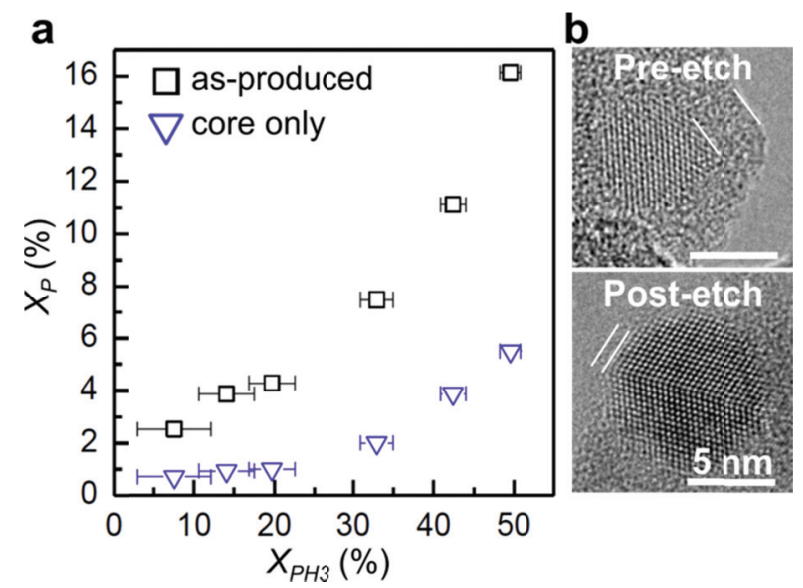

Figure 4. (a) Estimated atomic $\mathrm{P}$ concentration $\left(X_{P}\right)$ for as-produced samples and for samples after surface $\mathbf{P}$ had been removed. (b) Amorphous shell thickness of heavily P-doped SiNCs, which were fully oxidized in air ambient (Pre-etch), were compared to that of HF-vapor-etched one (Postetch) 